\title{
A Layer Assignment and Resource Reservation Scheme for Hierarchical Cell Structures
}

\author{
Roland Zander and Johan M. Karlsson \\ Department of Communication Systems, Lund University, Sweden ${ }^{\star}$ \\ \{rolandz, johan\}@telecom.1th.se
}

\begin{abstract}
To provide for a high QoS in a cellular network, each call should be assigned to its preferred cell and priority given to already ongoing calls. Herein, a combined speed-sensitive layer selection method and dynamic guard channel scheme, called CLARR, for use in hierarchical networks, is introduced. To reduce the complexity, partially the same speed estimation and measurement procedures are applied for both tasks. The main features of CLARR are the use of dwell time feedback control in the layer selection method and the grouping of calls depending on previous movement patterns. The measurements needed for making mobility predictions are performed separately for calls of different groups.
\end{abstract}

\section{Introduction}

In a cellular network, micro cells can out of efficiency reasons be inserted to cover hotspots such as airports and shopping malls. A micro cell causes usually less interference than a normal (macro) cell due to the low transmission power required for covering its smaller cell area. Another advantage with a hierarchical structure is the possibility to overflow traffic to alternative layers/cells. The layers are ordered according to cell size, where a layer consisting of small/large cells is denoted a lower/higher layer.

Cell/layer assignment is usually triggered by the location, carried service and/or mobility of the call. By assigning all calls to cells according to largest received signal strength, the average transmission power can be lowered, enabling a smaller reuse distance. The carried services must be considered due to differences in delay constraints and bandwidth demands [1]. Speed-sensitive methods assign slow/fast-moving calls to cells in the lower/higher layers, reducing the number of handovers, $E[H]$. There exist different procedures for estimating the subscriber speed. These include to quantify the power adjustments needed for power control [2], to continuously make subscriber location updates [3] and to measure the dwell times, $T_{d}$, in previous visited cells [4].

A subscriber finds it more annoying to have an ongoing call dropped than a new call blocked, meaning that prioritizing ongoing calls is a good idea for the operators. This can be accomplished by having some channels, called Guard Channels (GCs), exclusively reserved for handovers. The introduction of GCs reduces the handover dropping probabilities, $P_{d}$, at the expense of larger blocking probabilities, $P_{b}$, for new calls. The number of GCs can be either fixed or time-varying (dynamic).

* This work was supported by the Swedish Foundation for Strategic Research under the Personal

Computing and Communication (PCC) grant. 
In this paper, a combined speed-sensitive layer selection method and GC scheme for use in hierarchical cellular networks, called Combined Layer Assignment and Resource Reservation (CLARR), is introduced. In contrast to other layer assignment methods, CLARR does not only put the emphasis on the past speed of a call, but also on predicting its future $T_{d}$-values, using feedback control. A subscriber having previously moved with high/low speed will not necessarily have a short/long $T_{d}$ in its next cell. For example, the speed of a subscriber traveling by car to a major city will probably decrease due to speed limits. CLARR applies dynamic GCs to give higher priority to ongoing calls. The movements of previous calls are used for predicting the future whereabouts of the ongoing calls, determining the GC requirements in the different cells.

The outline of this paper is as follows. Section 2 includes a description of the CLARR method. The simulation model and the obtained numerical results can be found in section 3. Finally, conclusions are drawn in section 4.

\section{CLARR}

In CLARR, predictions of $T_{d}$ are used to determine to which cell/layer the ongoing calls shall be assigned (large/small predicted $T_{d} \Rightarrow$ lower/higher layer). When a handover is required, $T_{d}$ in the coverage area of the potential target cell belonging to the lowest covering layer is predicted. Previously measured $T_{d}$-values in this cell are used as input through a feedback procedure. By optimizing the assignment to the lower cellular layers where handovers are frequent, a handover rate reduction can be obtained.

Personalized subscriber treatment may be applied in the prediction procedure. Since this solution is of high complexity and mostly suffer from data shortage, $T_{d}$ of other subscribers ought to be considered. The accuracy of the predictions can be improved by grouping the $T_{d}$-values. This means that only measurements of calls of the same group as the call to be predicted are considered. In CLARR, the calls are grouped according to past speed since slow-moving subscribers usually have longer $T_{d}$-values than fastmoving subscribers. Further, the calls are grouped according to previous traveled paths (movement groups) since this helps anticipate route-dependent speed alterations (e.g., traffic jams) and traveled distances in the next cell (e.g., road paths).

When a call leaves the area of a cell belonging to its lowest covering layer and/or requires a handover, a mean of the most recent performed speed estimations (using an arbitrary speed estimation method) of the call is calculated. The subscriber speed in the cell is characterized by a number between 0 (slowest) and 1 (fastest), denoted $v$, where a value of 0.3 means that $70 \%$ of the previous calls in the cell had a higher speed. Exponential smoothing is applied to give the more recent data a larger weight. In (1), $V_{n}$ denotes the speed value obtained after $n$ iterations and $\alpha$ the smoothing parameter $(0 \leq \alpha \leq 1) . V_{n}$ is the parameter used to group the calls according to speed.

$$
V_{n}=\alpha V_{n-1}+(1-\alpha) v
$$

Following a call departure from the area of a cell of the lowest covering layer, its $T_{d}$-value is compared with those of all other calls measured in the cell. A percentile is calculated for $T_{d}$ and is sent to the location where $V_{n-1}$ for the call was calculated. There, the data are used for making predictions of $T_{d}$ for the next visited cell, $T_{n e x t}$, for calls 


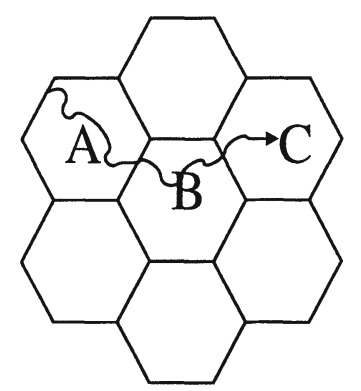

Fig. 1. Subscriber movements in a cellular network

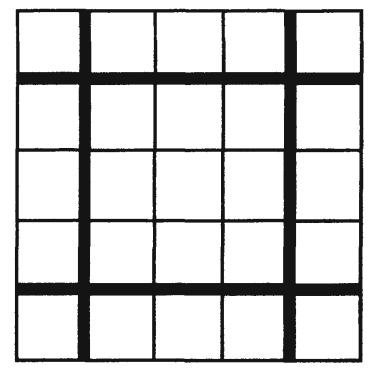

Fig. 2. Simulated network

of its movement and speed group. A movement group consists of calls having similar entry (identical previous cell) and exit points (identical next cell). $T_{n e x t}$ is calculated as a weighted mean of the most recent $T_{d}$-percentiles derived for calls of its group. For example, if the subscriber in Fig. 1 requires a handover from its current cell, C, its $T_{d^{-}}$ percentile in $\mathrm{C}$ is sent to its latest visited cell, B. There, it is considered when determining $T_{n e x t}$ for calls of a certain speed and movement group. Since the call arrived from A and then moved to $C$, the movement group in question is (A,C). The speed group is the group in which the call was placed following its handover from cell B.

When a call requires a handover, the $T_{\text {next }}$-value of its group is compared to a number of time-varying thresholds, equal to the number of cellular layers present in the region minus one. The call is assigned to the cell that corresponds to the number of thresholds that is larger/smaller than $T_{\text {next }}$ (high/low $T_{\text {next }} \Rightarrow$ lower/higher layer). If the load in any of the candidate cells is very high, the thresholds are adjusted so that fewer calls are assigned to the heavily loaded cells $[4,5]$.

CLARR includes a dynamic GC scheme measuring movement patterns of previous calls. The probability for a call residing in a certain cell to require a handover, $P_{h}$, and the probability for the handover to occur to cell $y, P_{h d}(y)$, where $y$ can be a cell of any hierarchical layer [6], are continuously measured. From these probabilities and the number of ongoing calls in the cell, $C$, the number of ongoing calls expected to require a handover to cell $y, G(y)$, can be calculated, see (2). By summation of all $G(y)$-values, an estimation of the current handover arrival rate in cell $y, G_{t o t}(y)$, is derived. Since all expected handover call arrivals to a cell will not occur simultaneously, the number of required GCs in cell $y$ is significantly less than $G_{t o t}(y)$. The number of allocated GCs is derived through a multiplication of $G_{t o t}(y)$ and a time-varying parameter $\psi(\psi<1)$.

$$
G(y)=C P_{h} P_{h d}(y)
$$

To improve the efficiency, separate measurements are performed for calls considered probable to have different movement patterns. Subscribers moving in opposite directions on a road have for instance completely different $P_{h d}$-values. Hence, the calls are divided into measurement groups, called source cell groups, according to latest visited cell. Since fast-moving subscribers often are more probable to require a handover than slow-moving subscribers, the calls in a source cell group are arranged into speed groups [6]. Here, the principles applied in the layer assignment part are reused. 


\section{Simulation Model and Numerical Results}

The network consists of two layers with micro and macro cells, respectively. It is out of simplicity reasons chosen to give the cells a quadratic shape although a hexagonal shape is much more realistic. The advantage with using quadratic cells is that it enables the area of each micro cell to be entirely covered by one macro cell. The lower layer consists of 81 micro cells in a $9 * 9$ ring topology with wrapped round edges. The micro cells are arranged into clusters of nine, $3 * 3$, where a macro cell provides coverage over the entire cluster area, see Fig. 2. A thin line corresponds to a border between two micro cells and a thick line to a border between both adjacent micro and macro cells.

The number of channels in a micro/macro cell is set to 20 and 60, respectively, and the call length is exponentially distributed (mean of 10 minutes). A new call is assigned to a micro cell or overflowed to the overlapping macro cell. The layer assignment thresholds are adjusted to achieve similar load ratios between the layers for all scenarios. A handover is attempted at the instant a call crosses a cell border. In case of a handover failure, the call is immediately dropped. Every single call is assigned an uniformly distributed number between 1 and 10, indicating the subscriber speed, through a randomization process at call arrival. This subscriber speed value is multiplied with a speed constant, unique for its current covering micro cell, resulting in a value describing how fast it is possible for the subscriber to move in its current cell. The mean dwell time in the covering micro cell is derived through multiplication between the inverse of this number and the distance to be traveled by the subscriber. The latter parameter indicates how much the subscribers tend to move around in the cell. This distance measure is dependent on the entry and exit points of the subscriber in the cell. The subscriber speed is estimated from past $T_{d}$-values mainly due to the low modeling complexity of this technique, but CLARR can operate under an arbitrary speed estimation procedure.

First, CLARR is compared to a reference method with identical features except for the division of calls into movement groups (set 1). The bandwidth reservation and load balancing features are discarded. Four different scenarios (SCs), I-IV in Table 1, are investigated. The $T_{d}$-distribution and the speed constants applied for the different micro cells separate the SCs. $T_{d}$ is assumed to be either uniformly or exponentially distributed. Three different parameter settings for the cell speed parameter are used, namely 1 for all cells, 2 for all cells, and 0.8 for $1 / 3$ of the cells and 1.1 for the remaining $2 / 3$ of the cells. When a call leaves its covering micro cell, it moves to each of the adjacent micro cells in a non-diagonal direction with an equal probability, namely $1 / 4$.

In simulation set 2 , it is investigated how beneficial it is to apply load balancing in CLARR. Still, the bandwidth reservation feature is discarded. The reference method is identical except for the load balancing feature. Two SCs are investigated (I and II). Cells with a momentary number of available channels smaller than a threshold, $T_{\text {load }}$, are classified as being heavily loaded. If a micro/macro cell is heavily loaded at the time of a call arrival and its overlapping macro/micro cell is not, the threshold determining to which cell the call should be assigned is increased/reduced. The size of the reduction/increase, $\Delta Q$, is derived through subtracting $T_{\text {load }}$ by the number of available channels in the heavily loaded cell, $N_{a v}$, and then multiply the result by a constant, $C$.

Simulation set 1 and 2 are investigated for three different traffic loads. The call failure probability, $P_{f}$, is the percentage of calls that are either blocked or dropped. In Table 3 , 
Table 1. Simulation scenarios (SCs)

\begin{tabular}{|c|c|c|c|}
\hline$S C$ & $T_{d}$-distribution & $P_{h d}$ & Cell Speed \\
\hline I & Uniform & $P_{1-4}=1 / 4$ & $1 / 1 / 1$ \\
\hline II & Uniform & $P_{1-4}=1 / 4$ & $2 / 2 / 2$ \\
\hline III & Uniform & $P_{1-4}=1 / 4$ & $0,8 / 1,1 / 1,1$ \\
\hline IV & Exponential & $P_{1-4}=1 / 4$ & $1 / 1 / 1$ \\
\hline V & Uniform & $P_{1-3}=1 / 3, P_{4}=0$ & $1 / 1 / 1$ \\
\hline
\end{tabular}

Table 2. Handover directions (hd)

\begin{tabular}{|c|c|}
\hline$h d$ & Explanation \\
\hline 1 & Micro cell straight ahead \\
\hline 2 & Back to the latest visited cell \\
\hline 3 & Micro cell to the left \\
\hline 4 & Micro cell to the right \\
\hline
\end{tabular}

Table 3. Numerical results for simulation sets 1 and 2

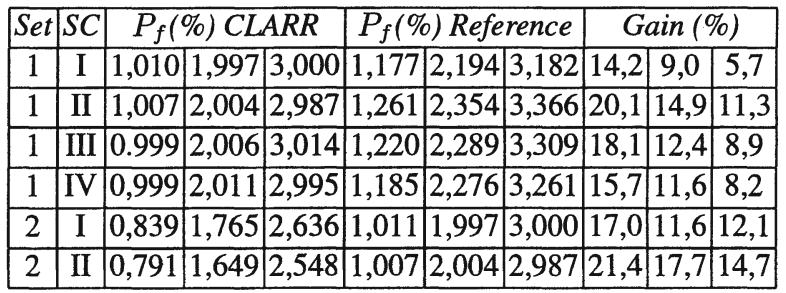

$P_{f}$ is shown for the CLARR and reference methods. The gain column shows the gain in percent obtained from dividing the calls into movement groups (set 1) or introducing load balancing (set 2). Further, $E[H]$ is also marginally larger for the reference scheme.

The introduction of movement groups increase the accuracy of the obtained speed estimations when using $T_{d}$ as a speed indicator, because calls of the same group have had similar straight path distances. The obtained gain of the grouping feature is larger at low traffic load because the $T_{d}$-predictions used for layer assignment only matters if there are available channels in the covering cells of both layers. Further, the gain is increased in case of a larger $E[H]$, see SC II. For every performed handover, a layer assignment selection is necessary. Thus, the importance of making accurate $T_{d}$-predictions increases with the number of handovers. Since the grouping feature places calls arriving from different cells into separate groups, differences in cell speed constants also increase the obtained gain, see SC III. When $T_{d}$ is exponentially distributed (SC IV), it becomes more difficult to make accurate $T_{d}$-predictions. However, these difficulties seem to affect both the CLARR and the reference method in similar ways. The higher gain for SC IV in comparison with SC I can probably be explained by the larger $E[H]$ that appears when an exponentially distributed $T_{d}$ is applied.

Introduction of load balancing in the CLARR method significantly reduces $P_{f}$, see set 2 in Table 3. The larger gain obtained for SC II is a result of an increased $E[H]$. However, it is somewhat difficult to make a comparison between the SCs because the speed threshold adjustments are not optimized but identical for both SCs. When the traffic load increases, the obtained gain is reduced due to a more evenly distributed momentary load between overlapping micro and macro cells.

In set 3 , the efficiency of the complete CLARR method is investigated. The reference method is identical except for the division of calls into movement and source cell groups. The parameter separating the two investigated SCs, I and V, is the probability for a call 
Table 4. Numerical results for simulation set 3

\begin{tabular}{|c|c|c|c|c|c|c|}
\hline$S C$ & $\begin{array}{c}P_{b}\left(10^{-2}\right) \\
\text { CLARR }\end{array}$ & $\begin{array}{c}P_{b}\left(10^{-2}\right) \\
\text { Reference }\end{array}$ & Gain (\%) & $\begin{array}{c}P_{d}\left(10^{-4}\right) \\
\text { CLARR }\end{array}$ & $\begin{array}{l}P_{d}\left(10^{-4}\right) \\
\text { Reference }\end{array}$ & Gain (\%) \\
\hline $\mathrm{I}$ & 1,579 & 2,129 & 25,8 & 8,34 & 8,75 & 4,7 \\
\hline $\mathrm{V}$ & 1,534 & 1,819 & 15,7 & 8,02 & 8,35 & 4,0 \\
\hline
\end{tabular}

to move to the coverage area of a specific adjacent micro cell, see Tables 1-2. For new calls, all adjacent micro cells are chosen with an equal probability.

The grouping feature significantly reduces $P_{b}$ and $P_{d}$, see Table 4 . Further, $E[H]$ is marginally smaller for CLARR. The obtained gain is reduced if the subscribers do not move back to their latest visited cell, see SC V. This alteration in $P_{h d}$ increases the accuracy of the movement predictions when applying the grouping feature, which ought to increase the obtained gain. Simultaneously, the differences in traveling distances in a cell are reduced. Thus, the difference in speed estimation efficiency between CLARR and the reference method is lowered. Since the obtained gain is smaller for SC V, this latter argument must have had a greater impact on the chosen simulation settings.

\section{Conclusion}

A combined speed-sensitive layer selection method and dynamic guard channel scheme for hierarchical cellular networks, called CLARR, has been introduced. Partially the same speed estimation and measurement procedures are applied for both tasks. Cell dwell time predictions together with the momentary cell loads are used as input to the layer assignment procedure. The number of guard channels to be allocated is determined by the number of ongoing calls in adjacent cells and their predicted movement patterns. To improve the accuracy of the predictions performed in the CLARR method, the calls are grouped according to previous movements. It was shown using simulations that a substantial gain can be obtained through its load balancing and grouping features.

\section{References}

1. J. Ben-Othman and F. Valois. Multiservice Allocation in Hierarchical Cellular Networks (MAHCN). IEEE Int. Symposium on Computers and Communication 1999, pp. 80-86, 1999.

2. S. Zhicum, W. Ying, Z. Ping and L. Baolin. Layer Selection Method using Power Control in Hierarchical Cellular Systems. IEEE VTC 2001, vol. 4, no. 4. pp. 2407-2411, 2001.

3. Y. Zhao. Standardization of Mobile Phone Positioning for $3 G$ Systems. IEEE Communications Magazine, vol. 40, no. 7, pp. 108-116, 2002.

4. K. Shum and C. W. Sung. Fuzzy Layer Selection Method in Hierarchical Cellular Systems. IEEE Transactions on Vehicular Technology, vol. 48, no. 6, pp. 1840-1849, 1999.

5. L. Jorguseski and D. Sparreboom. HCS: Decision Procedure for an Interlayer Handover. IEEE VTC 1999, vol. 3, pp. 1496-1500, 1999.

6. R. Zander and J. M. Karlsson. A Measurement-Based Dynamic Guard Channel Scheme for Handover Prioritization in Cellular Networks. 2nd International IFIP-TC6 Networking Conference "Networking 2002", pp. 1245-1251, 2002. 